\title{
Competencias profesionales e Industria 4.0: análisis exploratorio para ingeniería industrial y administrativa en Medellín*
}

\author{
Iván Darío Rojas Arenas** \\ Elizabeth Jiménez Medina*** \\ René Yepes Callejas*****
}

Recibido: 20-07-2020

Aceptado: $21-07-2020$

\begin{abstract}
Citar como: Rojas-Arenas, I. D., Jiménez-Medina, E. y Yepes-Callejas, R. (2021). Competencias profesionales e Industria 4.0: análisis exploratorio para ingeniería industrial y administrativa para en Medellín. Revista Interamericana de Investigación, Educación y Pedagogía, 14 (2), 169-194. https:// doi.org/10.15332/25005421.6299
\end{abstract}

\section{Resumen}

La formación por competencias implica un reto para las universidades en el contexto actual, en medio de la revolución que plantea la Industria 4.0 en los sistemas económicos. En este artículo se hace un análisis de las competencias requeridas en los programas de Ingeniería Industrial y Administrativa en la ciudad de Medellín de cara a este fenómeno. Se planteó una metodología exploratoria con enfoque mixto, en la que se aplicaron encuestas

\footnotetext{
* Artículo de investigación que presenta los resultados de un proyecto patrocinado por la Institución Universitaria Pascual Bravo de la ciudad de Medellín.

** Ingeniero industrial; especialista en Logística Integral; especialista en Gerencia Educativa; magíster en Gestión de la Innovación Tecnológica, Cooperación y Desarrollo Regional; doctorando en Pensamiento Complejo. Integrante grupo de investigación QUALIPRO y docente del programa de Ingeniería Industrial en la Institución Universitaria Pascual Bravo, Medellín, Colombia. Correo electrónico: ivan.rojasar@pascualbravo.edu.co ORCID: https://orcid.org/0000-0002-9300-3176po

Google Scholar: https://scholar.google.com/citations?user=HtlUklcAAAAJ\&hl=en CVLAC: https://scienti.minciencias.gov.co/cvlac/visualizador/generarCurriculoCv.do?cod_rh=0001607758

***. Magíster en Gestión Tecnológica; especialista en Formulación y Evaluación de Proyectos; ingeniera y administradora. Integrante grupo de investigación QUALIPRO y docente programa de Ingeniería Administrativa de la Institución Universitaria Pascual Bravo, Medellín, Colombia.

Correo electrónico: e.jimenezme@pascualbravo.edu.co

ORCID: https://orcid.org/0000-0002-5887-0872

Google Scholar: https://scholar.google.com/citations?user=uAtKpUEAAAAJ\&hl=es

CvLAC: https://scienti.minciencias.gov.co/cvlac/visualizador/generarCurriculoCv.do?cod_rh=0001458825

****. M. SC. y candidato a Ph. D. en Gestión de Tecnología e Innovación. Investigador del Centro de Estudios en Economía Sistémica (ECSIM) y docente en la Maestría en Gestión de Tecnología e Innovación de la Universidad Pontificia Bolivariana, Medellín, Colombia.

Correo electrónico: edgar.yepesc@upb.edu.co

ORCID: https://orcid.org/0000-0002-2398-0852

Google Scholar: https://scholar.google.com/citations?user=T_rSCOUAAAAJ\&hl=es\&oi=ao

CVLAC: https://scienti.minciencias.gov.co/cvlac/visualizador/generarCurriculoCv.do?cod_rh=0000268429
} 
en IES que ofrecían los programas antes mencionados, y empresas relacionadas con la Industria 4.0; asimismo, se realizó una consulta con orientación prospectiva (Delphi) a expertos en esta temática. La información secundaria se obtuvo de bases de datos a partir de una revisión sistemática de literatura y de la consulta en sitios web de distintas universidades de Medellín. Los resultados obtenidos muestran la importancia de flexibilizar los currículos y propender por la formación integral en ingeniería industrial y administrativa.

Palabras clave: competencias profesionales, Industria 4.0, formación por competencias, prospectiva, ingeniería, sistema educativo.

\title{
Professional Competencies and Industry 4.0: An Exploratory Analysis for Industrial and Administrative Engineering in Medellin
}

\begin{abstract}
The implementation of competency-based training is a challenge for universities in the current context, in the midst of the revolution in economic systems brought about by Industry 4.0. This article analyzes the competencies required in the industrial and administrative engineering programs in Medellin in face of this phenomenon. An exploratory methodology with a mixed approach was proposed, with surveys applied in higher education institutions that offered the aforementioned programs and companies related to Industry 4.0. In addition, a prospective-oriented consultation (Delphi) was carried out with experts on this subject. Secondary information was obtained from databases based on a systematic review of literature and the consultation on websites of different universities in Medellin. The results obtained show the importance of making curricula more flexible and promoting comprehensive training in industrial and administrative engineering.
\end{abstract}

Keywords: professional competencies, Industry 4.0, competencybased training, foresight, engineering, educational system. 


\section{Competências profissionais $e$ indústria 4.0: análise exploratória para engenharia industrial e administrativa para Medellín}

\section{Resumo}

O treinamento baseado em competências é um desafio para as universidades no contexto atual, em meio à revolução nos sistemas econômicos provocada pela indústria 4.0. Neste artigo, fazemos uma análise das competências necessárias nos programas de Engenharia Industrial e Administrativa da cidade de Medellín em vista desse fenômeno. Foi proposta uma metodologia exploratória com abordagem mista e desenvolveram-se pesquisas nas Instituições de Educação Superior (IES) que ofereciam os programas mencionados acima e em empresas relacionadas à Indústria 4.0. Além disso, foi realizada uma consulta com uma orientação prospectiva (Delphi) com especialistas nesse assunto. Informações secundárias foram obtidas de bancos de dados baseados em uma revisão sistemática da literatura e consultas em sites de várias universidades de Medellín. Os resultados obtidos mostram a importância de tornar os currículos mais flexíveis e de promover um treinamento abrangente em Engenharia Industrial e Administrativa.

Palavras-chave: competências profissionais, Indústria 4.0, treinamento por competências, previsão, engenharia, sistema educacional.

\section{Introducción}

Es evidente como el marcado cambio tecnológico que estamos viviendo impacta los sistemas económicos, productivos y educativos en el mundo. Actualmente los procesos de difusión de conocimiento están mediados por herramientas tecnológicas que sirven 
de apoyo en los modelos de aprendizaje (González, Berdugo y Martigo, 2017). La universidad tradicional se está quedando corta para afrontar los retos que supone la Industria 4.0 (14.0). En este contexto se hace pertinente hablar del concepto de competencia, y más aun lo que debe suponer la formación por competencias en educación superior y las metodologías de enseñanza-aprendizaje empleadas, de manera que los resultados obtenidos en este proceso sean pertinentes para el sector (Valdivieso, Montoya y Sánchez, 2018).

Según Estrada (2012), el concepto de competencia está determinado por un discurso empresarial que comprende aspectos como la capacidad de gestionar, el trabajo en equipo, la inteligencia emocional y el autoaprendizaje. Asún, Zúñiga y Ayala (2013) plantean que los modelos de formación por competencias hacen más efectivo el proceso de enseñanza-aprendizaje, ajustando planes de estudio y metodologías a necesidades del contexto, de manera que, como afirman Zúñiga, Leitón y Naranjo (2014), se adquieran habilidades cognitivas integrales. Zapata (2015) aporta a la discusión cuando comenta que la formación por competencias busca que los logros académicos se traduzcan en herramientas para pensar y actuar en el mundo, mientras que para Schlünzen Junior y colaboradores (2019) los currículos diseñados por competencias sobrepasan el modelo centrado en lo cognitivo.

Salcines y colaboradores (2018) tienen una visión interdisciplinar de este concepto, la cual incluye valores, habilidades y actitudes requeridas para llevar a cabo una labor, mientras que Cayambe y su equipo de investigadores (2018) definen la formación por competencias como un proceso continuo a lo largo de la vida que no depende de un título.

Álvarez, De Prada y Pino (2018) analizan la necesidad de evaluar los procesos formativos en educación superior a partir de la medición del impacto de las prácticas empresariales, entendiendo que existe una brecha entre academia y sector productivo que impide que los planes de estudio se ajusten a las necesidades de la industria. Esto lo complementa Rojas y colaboradores (2020), quienes 
realizan un estudio comparativo entre la precepción del proceso de formación por competencias teniendo en cuenta tres actores fundamentales: empresas, docentes y estudiantes, confirmando la diferencia en la percepción de los actores analizados al confrontar objetivos de formación de las universidades y necesidades de talento humano de las empresas.

Según Vera, Álava y Basurto (2019), el objetivo fundamental de la universidad debe ser formar profesionales con capacidad de aprender constantemente y adaptarse a los cambios, lo cual implica una transformación profunda en los modelos educativos. Ronquillo, Cabrera y Barberán (2019) convergen en lo anterior cuando exponen que la universidad ha tenido que evolucionar debido a cambios de orden económico, político y social, lo que ha generado la transición hacia modelos de formación por competencias, donde la universidad debe contar con planes de estudio flexibles, para lo cual necesita estar en contacto continuo con la industria y la sociedad. Según Neri y Hernández (2019), la competencia implica tanto aplicación de saberes como la posición que se asume en una organización para solucionar de forma eficiente problemáticas relativas al cargo que se desempeña. El reto: la formación en competencias para la 14.0.

Así las cosas, se plantea la necesidad de analizar la pertinencia de los procesos de formación en las instituciones de educación superior bajo el contexto actual de la 14.0. Si algo parece claro, es que los modelos de educación tradicionales muestran señales de obsolescencia frente al objetivo de formar profesionales integrales y con las competencias requeridas para enfrentarse a un mundo globalizado. La universidad tradicional, pegada a viejos parámetros de medición de la calidad educativa, está evolucionando hacia esquemas más flexibles, dinámicos y ajustados a una realidad que, en muchas ocasiones, era ajena a las aulas de clase. En este sentido, las competencias requeridas por un profesional están cambiando de forma radical, pues no se trata solo de saberes técnicos o acumulación de contenidos curriculares. La 14.0, más que títulos, requiere capacidades aplicadas en un contexto cambiante (Rentería et ál., 2019); asimismo, la forma como se desarrollan 
dichas competencias obedece a distintas metodologías de formación (Hernández, Restrepo y Rojas, 2020).

La 14.0, apalancada en temas como la transformación digital, automatización, robótica, internet de las cosas, big data o la inteligencia artificial, plantea retos, oportunidades y amenazas para las instituciones de educación superior (IES), que deben ajustarse a una demanda empresarial que requiere de personas formadas en competencias claves. La cuarta revolución industrial está impulsada por el capital humano, el cual es necesario para llevar a cabo de manera exitosa la incorporación y desarrollo de nuevas tecnologías, así que los países que tengan la capacidad de gestionar de forma adecuada sus sistemas educativos tendrán ventaja para liderar este cambio. Lo anterior presupone entender que las competencias en este nuevo entorno $-\mathrm{y}$, por ende, la formación por competencias - deben ser entendidas de manera distinta. Pérez (2016), por ejemplo, menciona competencias tales como adaptación al cambio, habilidades sociales, resolución de problemas complejos, creatividad, toma de decisiones y autoaprendizaje. Por su parte, Marcos y Martín (2016) entienden que más que habilidades específicas/técnicas, se requiere de personas con la capacidad de usar de forma eficiente la información con la que se cuenta.

Para Rivera (2016) la diversificación económica depende de la cualificación profesional, ya que una economía flexible y competitiva requiere capital humano con capacidad de adaptarse al mercado y aplicar del conocimiento. Fernández (2017) opina que esta nueva revolución tendrá grandes implicaciones en temas laborales, dados los cambios en las competencias y los nuevos mecanismos de relacionamiento a nivel empresarial. Samanes y Martínez (2018) aducen que la Cuarta Revolución Industrial está transformando no solo los sistemas productivos y la manera cómo hacemos las cosas, sino también la forma de percibirnos como seres humanos.

Pedroza (2018) habla de la reinvención de los sistemas educativos que permita la formación de capital humano apto para desempeñarse en el marco de los nuevos sistemas productivos mediados, mientras que Cedrola (2018) analiza cómo el trabajo del futuro va 
a estar asociado con aspectos como la capacidad de adaptación al cambio, la flexibilidad, el manejo efectivo de entornos virtuales y la medición de resultados.

Para el Banco Interamericano de Desarrollo (BID, 2018) algunas tendencias que marcarán el futuro del trabajo en América Latina son la revolución tecnológica y el envejecimiento demográfico; además, habla de la dificultad que tiene la región en temas de absorción tecnológica y las barreras que existen para la innovación, así como las problemáticas que se pueden generar con la creciente pérdida de empleos en muchas industrias y la precarización. Esta relación entre destrucción de empleos e 14.0 la analiza también Maiedah (2018) cuando habla del desempleo tecnológico: Para el autor, la demanda laboral de personas con competencias nuevas y más avanzadas hará obsoletas las habilidades valoradas en el mercado tradicional.

Meller y Salinas (2019) hablan de la necesidad de capital humano avanzado para la 14.0 , y de cómo los programas de pregrado en ingeniería y los de posgrados en ciencias básicas a nivel doctoral son fundamentales para la generación de nuevo conocimiento y el desarrollo de tecnologías que permitan competir a los países, dado que, en regiones como América Latina, la fuerza laboral está poco especializada y existe un distanciamiento entre el sector empresarial y la academia. Esto va en contravía de la tendencia que se da en algunos países desarrollados, en donde la industria y las universidades trabajan de forma colaborativa, lo que, sumado a la acelerada velocidad de los avances tecnológicos, ocasiona serias dificultades para la adaptación tecnológica en regiones menos desarrolladas.

\section{Metodología}

La presente investigación es de tipo exploratoria, prospectiva con enfoque mixto. El propósito fue reconocer las competencias que empiezan a ser demandadas en el entorno empresarial de la ciudad 
de Medellín para hacer posible la transformación digital hacia la 14.0, y la disposición de las universidades locales para atender dicha demanda.

El trabajo inició con la búsqueda de un marco de referencia sobre tipos de competencias que se han asociado con las actividades de las empresas bajo la 14.0. Para ello, se hizo una revisión sistemática de literatura, bajo un protocolo que consideró diferentes aspectos, como definición de criterios de inclusión y de exclusión, selección de términos de búsqueda, identificación de bases de datos académicas, desarrollo de las búsquedas en bases de datos, sistematización y evaluación de resultados, selección de resultados sobresalientes, realización de observaciones. La búsqueda arrojó más de 50 fuentes bibliográficas para la construcción de un marco de referencia sobre competencias asociadas a la 14.0, de las cuales se seleccionó un total de 28.

Las categorías de análisis fueron los conceptos de competencia, formación por competencias e Industria 4.0 en la educación. La información primaria se obtuvo de la manera como se explica a continuación:

- Entrevistas a directivos de IES públicas y privadas de Medellín que ofrecen programas de Ingeniería Industrial o afines, e Ingeniería Administrativa, con base en un cuestionario que incluyó preguntas sobre modelo pedagógico, conocimiento de temáticas de la 14.0, enfoques curriculares, competencias y asignaturas relativas a la 14.0, líneas de profundización e investigación, relacionamiento con la industria, planta docente e infraestructura. En la misma entrevista se hizo un análisis FLOR (fortalezas, limitaciones, oportunidades y riesgos).

- Encuesta basada en Delphi' con docentes y representantes de distintas IES que tuvieran conocimientos sobre temas de la 14.0 (25 en total). La selección se hizo con base en criterios como: experiencia y formación académica relacionada con la temática, dirección de grupos de investigación o programas de 
Ingeniería Industrial o Administrativa, haber liderado procesos de formación en competencias 4.0, tener roles directivos como decanaturas, coordinaciones académicas o de docencia. Estas personas representan a 13 IES públicas y privadas de Medellín.

- Encuesta basada en Delphi con expertos del sector empresarial en temáticas de la 14.0. Se formó un panel con 24 expertos radicados en Medellín, con amplios conocimientos en implementación de desarrollos en big data, analítica, inteligencia artificial, IOT (Internet of Things), realidad virtual o aumentada, incluyendo directivos de áreas de tecnología, innovación y gestión humana de empresas con experiencia en este tipo de aplicaciones. También participaron consultores y personas de entidades de CTI que apoyan procesos de transformación digital. El objetivo fue recopilar información que ayudara a identificar las necesidades de competencias y formación del talento humano en Medellín frente a la 14.0.

- Entrevistas de profundización en empresas (siete en total), en las que se indagaron las competencias requeridas por la industria, las tecnologías aplicadas de la 14.0, tipo de profesionales que buscan y lo que esperan de las universidades en términos de formación, en carreras como Ingeniería Industrial e Ingeniería Administrativa. La selección inició con una búsqueda de empresas de Medellín que trabajan temáticas relacionadas con la 14.0, lo que arrojó un listado de 80 empresas, las cuales fueron clasificadas en función de su actividad económica principal y el número de empleados. Con estos criterios, se identificó un grupo de 12 empresas representativas, ya fuera por pertenecer a actividades económicas donde se encontrarán múltiples empresas trabajando en 14.0 en Medellín, o por ser empresas con alta generación de empleo.

De igual forma, se buscó información secundaria de las IES que ofrecen los programas de Ingeniería Industrial, y afines e Ingeniería Administrativa de la ciudad de Medellín, tomando como elementos de análisis: la duración del programa, las asignaturas y el porcentaje 
por áreas de formación, la modalidad, el perfil ocupacional y profesional y las líneas de profundización. Dicha información fue obtenida de las páginas web de cada institución.

Con los resultados obtenidos mediante la aplicación de las entrevistas y tras sintetizar un catálogo de competencias pertinentes para la 14.0, se hizo una comparación de competencias ofrecidas por universidades y requeridas por empresas, para lo cual se ejecutaron dos encuestas simultáneas basadas en el enfoque Delphi, una a personal experto de las universidades y otra a especialistas de las empresas, para comparar el juicio de dichos expertos respecto a los plazos en los que se demandarán las competencias, y los plazos para que las universidades puedan ofrecerlas.

\section{Resultados}

Como punto de partida, se tomó un conjunto de competencias definidas en la revisión bibliográfica: manejo de lenguajes de programación, manejo efectivo de entornos virtuales, capacidad innovadora, flexibilización del aprendizaje, capacidad de autoaprendizaje, interdisciplinariedad, capacidad de usar de forma eficiente la información con la que se dispone, capacidad de procesar y analizar los enormes volúmenes de información, medición de resultados a partir de indicadores, pensamiento analítico, pensamiento multidisciplinario y crítico, pensamiento complejo, resolución de problemas complejos, liderazgo, toma de decisiones, adaptación al cambio, capacidad de adaptarse al mercado y generar valor agregado a partir de la aplicación del conocimiento, habilidades sociales, inteligencia emocional, creatividad. Se puede observar cómo entre estas competencias se encuentran muchas de las llamadas competencias blandas. Con base en la información secundaria obtenida de las páginas de las IES y la información primaria tomada de las entrevistas, se construyó una matriz de competencias. De igual forma, se sistematizó la información de las entrevistas realizadas en empresas para definir competencias comunes (tabla 1). 
Tabla 1. Matriz de competencias IES y empresas.

\begin{tabular}{|c|c|}
\hline \multicolumn{2}{|c|}{ Competencias comunes planteadas por IES y empresas } \\
\hline \multicolumn{2}{|c|}{ STEM } \\
\hline \multicolumn{2}{|c|}{ Desarrollo de aplicaciones matemáticas con el uso de software específicos } \\
\hline \multicolumn{2}{|c|}{ Manejo efectivo de entornos virtuales } \\
\hline \multicolumn{2}{|c|}{ Capacidad innovadora } \\
\hline \multicolumn{2}{|c|}{ Actuar como percutor de innovación (disparador de ideas y motivador) } \\
\hline \multicolumn{2}{|c|}{ Implementar proyectos de $\mathrm{I}+\mathrm{D}+\mathrm{i}$} \\
\hline \multicolumn{2}{|c|}{ Inglés } \\
\hline \multicolumn{2}{|c|}{ Liderazgo } \\
\hline \multicolumn{2}{|c|}{ Competencias afines (similares) entre IES y empresas } \\
\hline Competencias IES & Competencias empresas \\
\hline Manejo de lenguajes de programación & Capacidad de técnicas de programación \\
\hline Flexibilidad del aprendizaje & Flexibilidad \\
\hline $\begin{array}{c}\text { Pensamiento analítico y crítico, pensamiento } \\
\text { multidisciplinario }\end{array}$ & $\begin{array}{c}\text { Aprendizaje analítico, aprendizaje continuo y } \\
\text { permanente, pensamiento crítico }\end{array}$ \\
\hline Aplicar capacidades en un contexto cambiante & Adaptación al cambio \\
\hline Inteligencia emocional & Relacionamiento, inteligencia emocional \\
\hline Comunicación efectiva & Comunicación asertiva \\
\hline \multicolumn{2}{|c|}{ Competencias no comunes entre IES y empresas } \\
\hline Competencias IES & Competencias empresas \\
\hline Trabajo en equipo & Visión sistémica \\
\hline Toma de decisiones & Pensamiento complejo \\
\hline $\begin{array}{l}\text { Adaptación al mercado y generación de } \\
\text { valor agregado a partir de la aplicación de } \\
\text { conocimiento }\end{array}$ & Pensamiento sistémico \\
\hline Resolución de problemas complejos & Aprendizaje acelerado \\
\hline Creatividad & Actitud hacia el cliente: orientación al cliente \\
\hline Aplicación de tecnologías en las organizaciones & Disposición ética \\
\hline $\begin{array}{c}\text { Habilidades para trabajar bajo esquemas de } \\
\text { interdisciplinariedad }\end{array}$ & Formación humanística y de valores \\
\hline $\begin{array}{l}\text { Procesamiento y análisis de enormes volúmenes } \\
\text { de información }\end{array}$ & \multirow{4}{*}{ Disposición a disfrutar del fracaso } \\
\hline $\begin{array}{c}\text { Integración de tecnologías y soluciones de la } \\
\text { Industria } 4.0\end{array}$ & \\
\hline Entendimiento de la transformación digital & \\
\hline $\begin{array}{l}\text { Conocimiento de conceptos básicos de la } \\
\text { automatización y su aplicación }\end{array}$ & \\
\hline
\end{tabular}

Fuente: elaboración propia. 
Lo mostrado en la tabla 1 confirma lo dicho en un párrafo anterior en torno al tipo de competencias que se están requiriendo y las que pretenden brindar las IES en sus programas académicos de Ingeniería Industrial y Administrativa. Se observa la manera en que las competencias relacionadas con tecnología y ciencias exactas (STEM) se priorizan como pilares profesionales de cara a los retos de la 14.0. Asimismo, es interesante ver cómo los empresarios hablan de la formación en valores, la actitud y la ética.

En la entrevista a empresas se preguntó por el tipo de profesionales que buscan y lo que esperan de las IES en términos de formación. Entre las consideraciones respecto al tipo de profesionales, hubo énfasis en la necesidad de personas con características integrales, que trasciendan la especialización técnica. Se planteó la importancia que las personas tengan orientación a resultados, así como a la resolución de problemas, que incluye la capacidad para entender problemáticas, conceptualizar alternativas de solución y gestionarlas. También se relacionó dicha integralidad con un personal comprometido, con pensamiento crítico y perfil de liderazgo, y con una formación transversal, capaz de aportar de manera versátil en el desarrollo de múltiples soluciones. De acuerdo con lo expresado por los entrevistados, otras características deseables en las personas son la disposición a superar el miedo a lo nuevo, la capacidad para entender problemas y el sentido ético.

Por su parte, se reportaron necesidades de tipo técnico tales como:

- Conocimientos y habilidades matemáticas y de operaciones numéricas, estadísticas y en áreas afines, como el manejo avanzado de hojas de cálculo y otras herramientas de análisis matemático, estadístico y numérico.

- Competencias en diversas ramas de la ingeniería, con énfasis en tecnologías propias de la 14.0.

- Competencias para desarrollar la arquitectura de soluciones, incluyendo conocimiento en procesos, contextos 
de aplicación de las tecnologías, diseño y análisis de sistemas, y capacidad para modelar una empresa.

- Competencias específicas para las tecnologías propias de la 14.0, como conectividad en nube, gestión y análisis de datos, capacidad para configurar y operar dispositivos y equipos.

- Habilidades para desarrollo de software.

- Competencias relacionadas con la gestión de procesos y proyectos.

Frente a la pregunta que se formuló a las empresas sobre qué cambios esperan de las IES para adecuarse al nuevo contexto de la 14.0, se encontró:

- Se considera necesario cambiar a métodos de aprendizaje basados en la experiencia y práctica, que permitan a los estudiantes conocer la realidad de los entornos de aplicación de las tecnologías y conceptos. La educación dual fue considerada una estrategia prometedora en este sentido.

- Se considera que, sin dejar de entrenar adecuadamente a los estudiantes en asuntos técnicos y empresariales asociados a la 14.0, se requiere ir más allá, para formar personal con habilidades diferenciadoras. Esto implica que se desplieguen procesos formativos que den más fuerza a actitudes y valores.

- Se plantea la conveniencia de consolidar el relacionamiento empresa-universidad, para actividades que generen sinergias entre investigación, formación y extensión, aunque ello implique resolver barreras de ambos lados que han limitado su actividad colaborativa. 
- Se considera que la transformación que se está dando constituye una oportunidad para que las universidades asuman un rol visible como agentes de cambio, aportando nuevas competencias y también integrándose a redes de trabajo asociadas a iniciativas de transformación digital.

- Para que las universidades se conviertan en formadores de competencias y difusores de conocimiento idóneos para la transformación digital, será necesario que alcancen y mantengan una actualización tecnológica en herramientas disponibles, así como en conocimientos dominados por su cuerpo docente. Para esta condición es fundamental la participación de las empresas, que ayuden a la universidad a identificar y apropiar dichos elementos tecnológicos en el estado del arte.

- Se conceptuó que en el medio empresarial que está implementando activamente la 14.0 en Medellín empieza a replantearse el requisito de un título profesional específico como garantía para acceder a personas con las competencias adecuadas. Mencionando experiencias propias y de otras empresas reconocidas en la ciudad, se relataron prácticas de vinculación de personal para la 14.0 en las que se ha optado por no exigir un título profesional específico, e incluso se acepta personal sin ningún título, pero que pueda demostrar que ha adquirido competencias para laborar en esta área.

De la información obtenida de las IES a partir de las entrevistas realizadas y la búsqueda en sus páginas web, contrastada con la revisión bibliográfica y los resultados de las entrevistas a las empresas, se definieron unas competencias que se ubicaron en tres grupos: competencias del saber hacer, del saber y del ser (Ministerio de Educación Nacional [MEN], 2008), las cuales se muestran en la tabla 2. 
Tabla 2. Competencias para la 14.0.

\begin{tabular}{|c|c|c|}
\hline Competencia & Definición & Categoría \\
\hline $\begin{array}{c}\text { STEM (Science, } \\
\text { Technology, Engineering \& } \\
\text { Mathematics) }\end{array}$ & $\begin{array}{l}\text { Habilidad para entender y aplicar estas disciplinas en la } \\
\text { solución de problemas en contextos reales. }\end{array}$ & \multirow{5}{*}{ Saber hacer } \\
\hline $\begin{array}{c}\text { Aplicación de } \\
\text { herramientas tecnológicas } \\
\text { y lenguajes de } \\
\text { programación para la } \\
\text { solución de problemas en } \\
\text { contextos reales. }\end{array}$ & $\begin{array}{c}\text { Incluye el manejo de lenguajes de programación, } \\
\text { desarrollo de aplicaciones matemáticas mediante } \\
\text { software específicos, manejo de entornos virtuales } \\
\text { y demás temas orientados al uso de herramientas } \\
\text { tecnológicas y de programación para solucionar } \\
\text { problemas. }\end{array}$ & \\
\hline $\begin{array}{l}\text { Capacidad innovadora- } \\
\text { pensamiento innovador }\end{array}$ & $\begin{array}{c}\text { Ser un percutor de innovación, disparador de ideas, } \\
\text { capaz de involucrarse en proyectos innovadores, desde } \\
\text { la idea hasta la puesta en operación. }\end{array}$ & \\
\hline Trabajo en equipo & $\begin{array}{c}\text { Participar de forma activa en grupos, haciendo aportes } \\
\text { proactivos que permita la solución de problemáticas. } \\
\text { Implica interactuar con varias personas al tiempo y bajo } \\
\text { presión. }\end{array}$ & \\
\hline $\begin{array}{l}\text { Manejo de un segundo } \\
\text { idioma }\end{array}$ & Conocimiento de otro idioma, especialmente inglés. & \\
\hline $\begin{array}{l}\text { Aprendizaje constante y } \\
\text { en entornos flexibles de } \\
\text { formación }\end{array}$ & $\begin{array}{l}\text { Incluye capacidad de autoaprendizaje, flexibilidad en } \\
\text { el proceso de formación y adquisición permanente de } \\
\text { conocimientos de forma interdisciplinar. }\end{array}$ & \multirow[b]{2}{*}{ Saber } \\
\hline $\begin{array}{l}\text { Pensamiento holístico y } \\
\text { sistémico aplicado en la } \\
\text { integración de tecnologías } \\
\text { bajo el paradigma de } \\
\text { transformación digital. }\end{array}$ & $\begin{array}{l}\text { Incluye la integración de soluciones de la Industria } \\
\text { 4.0. En general, realiza integración de tecnologías, } \\
\text { además tiene entendimiento de la transformación } \\
\text { digital, conocimiento de los conceptos básicos de } \\
\text { la automatización y de la tecnología aplicada a las } \\
\text { organizaciones. }\end{array}$ & \\
\hline Pensamiento complejo & $\begin{array}{l}\text { Capacidad de entender un objeto de análisis a partir de } \\
\text { sus variables y las relaciones que se establecen entre } \\
\text { ellas. Incluye pensamiento analítico, multidisciplinario y } \\
\text { crítico, además resolución de problemas complejos. }\end{array}$ & \multirow{7}{*}{ Ser } \\
\hline Liderazgo y proactividad & $\begin{array}{c}\text { Capacidad de influir en el grupo de trabajo para el } \\
\text { logro de objetivos; de tomar la iniciativa y proponer } \\
\text { soluciones. }\end{array}$ & \\
\hline Toma de decisiones & $\begin{array}{c}\text { Determinación y elección oportuna entre las opciones } \\
\text { para resolver una situación. Implica capacidad } \\
\text { resolutiva bajo incertidumbre. }\end{array}$ & \\
\hline Adaptación al cambio & $\begin{array}{l}\text { Aceptar el cambio como una constante en las } \\
\text { organizaciones, adaptándose a nuevas dinámicas del } \\
\text { mercado y el entorno. }\end{array}$ & \\
\hline Inteligencia emocional & $\begin{array}{l}\text { Incluye habilidades sociales, comunicación efectiva y el } \\
\text { ser integral. }\end{array}$ & \\
\hline Creatividad & $\begin{array}{l}\text { Capacidad de analizar los problemas o circunstancias } \\
\text { desde diferentes puntos de vista, brindando soluciones } \\
\text { nuevas y originales. }\end{array}$ & \\
\hline $\begin{array}{l}\text { Resiliencia. Capacidad } \\
\text { de afrontar los problemas } \\
\text { y brindar soluciones } \\
\text { adecuadas. }\end{array}$ & $\begin{array}{c}\text { Incluye disfrutar del fracaso en los procesos que se } \\
\text { realicen de la Industria } 4.0 \text {. Disposición para sacar } \\
\text { adelante un proceso o proyecto, a pesar de las } \\
\text { dificultades. }\end{array}$ & \\
\hline
\end{tabular}

Fuente: elaboración propia. 
Las competencias anteriores comprenden un grupo que atiende aspectos propios de la 14.0, como lo son las STEM o el manejo de herramientas tecnológicas y de lenguajes de programación. También hay competencias que obedecen más a la integralidad del profesional, dado que están relacionadas con aspectos personales y no con conocimientos específicos.

Por último, se establecieron dos paneles de expertos. Uno con expertos de universidades en tecnologías de la 14.0 y directivos que han trabajado en el diseño y puesta en marcha de programas de educación superior, y en modelos de formación de competencias. El otro panel incluyó personal de empresas con experiencia en la implementación de soluciones de la 14.0, consultores con conocimiento técnico y de gestión en proyectos de esta área, y ejecutivos de instituciones que apoyan procesos de transformación digital en empresas.

Con cada panel se hizo una ronda de encuesta bajo aproximación Delphi, utilizando el catálogo de competencias presentado en la tabla 2. Se indagó el juicio de expertos respecto a cuánto tiempo podría tomar a las universidades ofrecer cursos u otras herramientas formativas orientadas a desarrollar las competencias identificadas. Por su parte, los expertos empresariales juzgaron el plazo en el que dichas competencias serían requeridas en el mercado laboral. Para ambos casos, se obtuvieron respuestas tendientes a plazos cortos, tanto para configurar una capacidad de oferta como para empezar a demandar las competencias. Se calculó el promedio aritmético de los plazos indicados por cada panel y para cada competencia, y de la diferencia entre el plazo promedio de oferta de las competencias y el plazo promedio de demanda de estas; así, cuando la brecha arroja un valor negativo, se interpreta como indicio de que la capacidad de respuesta de las universidades para formar las competencias, en promedio, podría ser lenta frente a la demanda. En cambio, una brecha positiva sería un indicio de una buena capacidad de respuesta.

Los resultados de este análisis prospectivo se muestran en las tablas 3,4 y 5 . 
Tabla 3. Diferencia entre demanda y oferta: competencias del saber.

\begin{tabular}{|c|c|c|c|}
\hline Competencia & $\begin{array}{c}\text { Años para } \\
\text { requerir } \\
\text { (demanda) } \\
\text { según } \\
\text { empresas }\end{array}$ & $\begin{array}{c}\text { Años para } \\
\text { disponibilidad } \\
\text { (oferta) según } \\
\text { universidades }\end{array}$ & $\begin{array}{c}\text { Brecha (plazo } \\
\text { demanda } \\
\text {-plazo oferta) }\end{array}$ \\
\hline $\begin{array}{c}\text { Aprendizaje constante y en entornos flexibles de } \\
\text { formación }\end{array}$ & 0.63 & 0.92 & -0.29 \\
\hline $\begin{array}{c}\text { Procesamiento y análisis de la información para la } \\
\text { toma de decisiones }\end{array}$ & 0.77 & 0.80 & $-0,03$ \\
\hline $\begin{array}{c}\text { Pensamiento holístico y sistémico aplicado en la } \\
\text { integración de tecnologías bajo el paradigma de } \\
\text { transformación digital }\end{array}$ & 1.00 & 1.08 & -0.08 \\
\hline
\end{tabular}

Fuente: elaboración propia.

Se reconoce que las tres competencias clasificadas como del saber muestran un valor negativo en el cálculo de brecha, lo cual sugiere que el plazo de formación de estas competencias podría retrasarse frente a su demanda. La brecha es más significativa para la competencia de Aprendizaje constante y en entornos flexibles de formación.

Tabla 4. Diferencia entre demanda y oferta: competencias del saber hacer.

\begin{tabular}{|c|c|c|c|}
\hline Competencia & $\begin{array}{c}\text { Años para } \\
\text { requerir } \\
\text { (demanda) } \\
\text { según } \\
\text { empresas }\end{array}$ & $\begin{array}{c}\text { Años para } \\
\text { disponibilidad } \\
\text { (oferta) según } \\
\text { universidades }\end{array}$ & $\begin{array}{c}\text { Brecha (plazo } \\
\text { demanda } \\
\text {-plazo oferta) }\end{array}$ \\
\hline STEM & 1.16 & 0.74 & 0.42 \\
\hline $\begin{array}{c}\text { Aplicación de herramientas tecnológicas y } \\
\text { lenguajes de programación para la solución de } \\
\text { problemas en contextos reales }\end{array}$ & 0.64 & 0.80 & -0.16 \\
\hline \begin{tabular}{c} 
Capacidad innovadora - pensamiento innovador \\
\hline Trabajo en equipo
\end{tabular} & 0.80 & 0.88 & -0.08 \\
\hline Manejo de un segundo idioma & 0.54 & 0.40 & 0.14 \\
\hline
\end{tabular}

Fuente: elaboración propia. 
Para el caso de las competencias del saber hacer, se encuentran menos resultados negativos, lo cual supone que en este aspecto la capacidad de respuesta de las universidades puede ser mejor. Lo anterior puede explicarse por el hecho de que dicho grupo está constituido por las competencias relacionadas con asuntos técnicos, en los que las IES eventualmente pueden desplegar más rápidamente una capacidad, o tener algunas opciones de formación ya constituidas. Sin embargo, aún existe una diferencia negativa en tres competencias de este grupo, como es el caso del Manejo de un segundo idioma, que podrían constituir un cuello de botella frente a las necesidades de la demanda empresarial.

La situación que se presenta en las competencias del ser requiere de un análisis complejo, ya que se evidencia que todas las diferencias son negativas y en su mayoría con valores de brecha significativos (para cinco de las seis competencias, la brecha se encuentra en un rango entre -0.26 y -0.56).

Tabla 5. Diferencia entre demanda y oferta: competencias del ser.

\begin{tabular}{|c|c|c|c|}
\hline Competencia & $\begin{array}{c}\text { Años para } \\
\text { requerir } \\
\text { (demanda) } \\
\text { según } \\
\text { empresas }\end{array}$ & $\begin{array}{c}\text { Años para } \\
\text { disponibilidad } \\
\text { (oferta) según } \\
\text { universidades }\end{array}$ & $\begin{array}{c}\text { Brecha (plazo } \\
\text { demanda } \\
\text {-plazo oferta) }\end{array}$ \\
\hline Pensamiento complejo & 1.14 & 1.40 & -0.26 \\
\hline Liderazgo y proactividad & 0.70 & 0.78 & -0.08 \\
\hline Adaptación al cambio & 0.57 & 0.96 & -0.39 \\
\hline Inteligencia emocional & 0.66 & 1.26 & -0.60 \\
\hline Creatividad & 0.57 & 0.84 & -0.27 \\
\hline $\begin{array}{c}\text { Resiliencia - capacidad para afrontar los problemas } \\
\text { y brindar soluciones adecuadas }\end{array}$ & 0.64 & 1.20 & -0.56 \\
\hline
\end{tabular}

Fuente: elaboración propia.

Las tablas 3, 4 y 5 ayudan a reconocer las nuevas competencias en las que, tras contrastar las percepciones de los dos paneles de expertos, es posible que las universidades puedan ser oportunas en el desarrollo de una oferta innovadora. En este sentido, se destaca que para las competencias STEM y Trabajo en equipo el resultado de la comparación fue favorable, lo que significa que las universidades 
podrían adecuar su oferta formativa de manera oportuna, anticipándose incluso a los plazos de demanda. De acuerdo con esto, el cambio estaría sujeto principalmente a que las universidades tomen la decisión de activar los procesos de ajuste necesarios.

Tras resaltar las dos competencias anteriores, donde hay indicios de ventaja por parte de las universidades, se debe notar que las otras competencias analizadas (12 de un total de 14) están en una situación desfavorable, dado que el cálculo de brecha fue negativo. Esto sugiere un análisis riguroso, en donde se considere que el tiempo para configurar la oferta es mayor al plazo de la demanda, lo cual implicaría desarrollar acciones de ajuste bajo ruta crítica. Aunque la mayoría de las competencias del catálogo se encuentran en esta misma condición, merecerían atención especial: Aprendizaje constante y en entornos flexibles de formación, Manejo de un segundo idioma, Adaptación al cambio, Inteligencia emocional y Resiliencia, que corresponden a las competencias donde la brecha negativa fue de magnitud mayor, lo que debe interpretarse como indicios de una menor capacidad de respuesta de las IES.

Si se revisan los resultados para los tres grupos de competencias, se nota que en las del saber hacer hay resultados mixtos, en las del saber se calcularon brechas ligeramente negativas, mientras en las competencias del ser los valores de brecha son los más negativos. De aquí se pueden extraer señales que alerten sobre la mayor dificultad que podría constituir el desarrollo de las competencias del ser, especialmente en aspectos como la Resiliencia, Inteligencia emocional y Adaptación al cambio. Esta situación merecería ser evaluada por las universidades, pues este tipo de competencias fue indicado por las empresas como necesario para que los profesionales tengan perfiles integrales y ajustados a las nuevas exigencias que afronta el novedoso régimen tecnológico bajo el que empiezan a operar. 


\section{Conclusiones}

Hablar de competencias para la 14.0 implica comprender la manera en que una revolución de los sistemas productivos, mediada por el acelerado avance tecnológico, condiciona los requerimientos formativos de los profesionales en carreras como la Ingeniería Industrial y la Ingeniería Administrativa. Es importante entender que la transformación digital hacia la 14.0 no se limita a cambios locales y continuos en algunas tecnologías, sino que constituye un cambio de paradigma tecnoeconómico en los sistemas industriales del siglo XXI, que implica modificaciones profundas en la forma de pensar y en "los hábitos existentes en tecnología, economía, gerencia e instituciones sociales" (Pérez, 2004, p .30). Esta oleada de transformación obliga a todos los agentes, y en particular a las empresas y universidades, a repensar el rol del trabajador en el futuro próximo y, ligado a ello, los procesos de enseñanza vigentes. Los hallazgos de este estudio muestran nuevas competencias, así como competencias que, si bien ya se tenían identificadas como complementarias, ahora empiezan a ser reconocidas como fundamentales para el desempeño profesional.

El catálogo de competencias resultante de este trabajo es material de discusión para los estamentos universitarios, y en la medida que se vayan adoptando como parte de los perfiles a formar, será necesario que se revisen los modelos de formación. Las nuevas competencias requieren enfoques que trasciendan los esquemas formativos tradicionales, orientados a la transmisión de conocimientos, y que pueden alejar a los estudiantes del contexto real. Aunque es de vieja data la preocupación por renovar los patrones formativos en las universidades, la disrupción de la 14.0 hace más apremiante que las IES se comprometan en la adecuación y flexibilización de sus currículos y modelos formativos.

La I4.0 no solo implica cambios en las competencias técnicas requeridas por las empresas, sino que plantea una formación integral de los sujetos, quienes deben interactuar en las organizaciones aplicando los conocimientos específicos de su área de formación 
al tiempo que se relacionan en un medio particular; aspectos como la capacidad de aprender constantemente, la resiliencia o el liderazgo pueden ser más importantes que manejar un equipo o un software específicos.

El concepto de competencia tiene raíces en la tradición empresarial, como una forma de definir las capacidades, habilidades o destrezas que debe tener una persona para el adecuado desempeño de su labor; sin embargo, el concepto se vuelve complejo de abordar en un contexto de cambio de paradigma tecnoeconómico. Según este estudio, la disrupción de la 14.0 acarrea un nuevo perfil de demanda de competencias más diversificado y relacionado con un campo de actuación más amplio. De la mano con esto, se detectó un cambio de tendencia respecto a los requerimientos académicos exigidos al personal. Según los empresarios, hoy prefieren que una persona posea conocimientos y destrezas específicos relativos a su área de actuación así no posea un título universitario, lo cual generalmente requiere un periodo largo de estudio. Además, las organizaciones valoran mucho que la universidad se acerque a la industria, de manera que se generen programas más pertinentes con las realidades del medio.

Para el caso particular de Medellín, es evidente que las empresas tienen una visión distinta frente al proceso formativo respecto de las IES. Del análisis realizado, se observa que muchos programas y cursos aún se basan en modelos de transmisión de conocimiento orientados a los contenidos, enfocados en cumplir con la exposición de elementos de información incorporados en materiales académicos, lo cual no ha demostrado ser insuficiente para la formación integral de las personas. Las empresas, en cambio, se interesan más en la capacidad de resolver problemas en el entorno laboral, y ya no tanto en el título o el total de asignaturas que haya cursado un individuo durante su carrera universitaria. Esta observación señala una divergencia que merece ser revisada por universidades y tejido empresarial en conjunto, para evitar una falla sistémica debida a la suficiencia de los perfiles de talento humano que hoy están egresando, frente a los retos que se deben abordar en los nuevos ambientes de trabajo. 
La formación por competencias en la 14.0 impone un reto para las IES que pretenden una formación pertinente y que brinde soluciones reales a la sociedad. Aspectos como la flexibilidad de los planes de estudio, la disminución de los tiempos de formación o la posibilidad de acceder a certificaciones por competencias en cada etapa del proceso formativo son fundamentales frente a las exigencias del mercado laboral actual.

Esta investigación tuvo la fortaleza de haber contado con expertos en el objeto de estudio, tanto del sector empresarial como del educativo; fue muy importante para este análisis tener las apreciaciones de dos actores fundamentales en el proceso educativo. Asimismo, las competencias encontradas en la revisión bibliográfica muestran una relación cercana a las propuestas por empresarios e IES. Se podría decir que teóricamente se sabe hacia dónde deben apuntar los procesos formativos y qué busca el mercado laboral. Sin embargo, la realidad apunta a que aun estos procesos no están afinados y no responden totalmente a las necesidades de las organizaciones. Lo anterior se explica en aspectos como los altos costos y esfuerzos que implica la búsqueda, contratación y capacitación de personal nuevo de la 14.0 en las empresas, que fue una dificultad planteada de forma general por los voceros empresariales.

El tema de la formación integral se hace evidente cuando se analizan los resultados; las empresas y la sociedad no solo requieren personas con conocimientos técnicos, sino que además sean buenos ciudadanos, con valores éticos y morales, y con capacidades como la adaptación al cambio, la resiliencia, el liderazgo, el aprendizaje continuo y el trabajo en equipo.

Llama la atención la brecha que existe entre las necesidades del mercado laboral y la capacidad de las IES para cumplir con dichas necesidades. Es claro que, con modelos educativos poco flexibles, los resultados de formación no se obtienen en el tiempo adecuado, de manera que se facilite la inserción laboral. Esto podría incentivar a las empresas a buscar alternativas de formación interna, o reclutamiento de personal sin considerar títulos profesionales. Ante este riesgo para las entidades educativas, podrían considerarse 
programas académicos que brinden una formación por bloques o áreas que puedan certificarse, permitiendo a las personas acceder fácilmente a un trabajo.

El modelo de formación dual se plantea como una alternativa interesante. En el caso de Medellín, ya se cuenta con experiencias exitosas. Esta estrategia podría permitir a las IES validar sus procesos de enseñanza-aprendizaje en entornos reales, sin esperar que las personas se gradúen de un programa específico. Además, permitiría un acercamiento entre la industria y la academia, que está establecido como un propósito generalizado para las IES de esta ciudad.

\section{Referencias}

Álvarez, M., De Prada, E., y Pino, M. (2018). Estudio sobre la capacidad técnica y las competencias transversales desarrolladas en las prácticas externas universitarias. Revista Estudios Pedagógicos, 44(3), 137-155. https://doi.org/10.4067/S0718-07052018000300137

Asún, R., Zúñiga, C., y Ayala, M. (2013). La formación por competencias y los estudiantes: confluencias y divergencias en la construcción del docente ideal. Revista Calidad en la Educación, (38), 277-304. https:// doi.org/10.31619/caledu.n38.112

Banco Interamericano de Desarrollo (BID). (2018). El futuro del trabajo en América Latina y el Caribe. ¿Una gran oportunidad para la región? https://www.iadb.org/futurodeltrabajo

Cayambe, M., Filian, F., Argüello, L. y Rodríguez, N. (2018). Formación por competencias: reto actual de la universidad ecuatoriana. Didasc@lia: Didáctica y Educación, 9(1), 141-150. https://dialnet.unirioja.es/servlet/ articulo?codigo $=6595075$

Cedrola, G. (2018). Economía digital e Industria 4.0: reflexiones desde el mundo del trabajo para una sociedad del futuro. Revista Internacional y Comparada de Relaciones Laborales y Derecho del Empleo, 6(1), 261-297. 
Estrada, J. (2012). La formación por competencias y el mundo del trabajo: de la calificación a la empleabilidad. Revista de Salud Pública, 14(1), 98-111.

Fernández, J. (2017). El futuro del trabajo vs. El trabajo del futuro. Implicaciones laborales de la industria 4.0. Revista Jurídica de la Universidad de León, (4), 211-213.

González, K., Berdugo, N., y Martigo, A. (2017). Incidencia de los entornos virtuales de aprendizaje en la calidad de la educación superior, desde el contexto colombiano. Revista Interamericana de Investigación, Educación y Pedagogía (RIIEP), 10(2), 11-24. https://doi.org/10.15332/ s1657-107X.2017.0002.01

Hernández, K., Restrepo, K., y Rojas, I. (2020). Análisis a los métodos de formación utilizados en las escuelas de destrezas implementadas por las empresas para mejorar el rendimiento en sus plantas de producción. En E, Serna (ed.), Revolución en la formación y la capacitación para el siglo XXI. (pp. 527-537). Editorial Instituto Antioqueño de Investigación.

Maiedah, J. (2018). Industria 4.0, competencia digital y el nuevo sistema de formación profesional para el empleo. Revista Internacional y Comparada de Relaciones Laborales y Derecho del Empleo, 6(1), 164-194.

Marcos, E y Martín, M. (2016). Formación de profesionales para la empresa del siglo XXI. Ekonomiaz: Revista vasca de economía, (89), 174-193.

Meller, P y Salinas, B. (2019). Revolución tecnológica 4.0 y capital humano. Una mirada desde la minería. Beauchef Minería. Serie de estudios sobre minería, tecnología y sociedad. Universidad de Chile.

Ministerio de Educación Nacional (MEN). (2008). Diseño y ajuste de programas de formación para el trabajo bajo el enfoque de competencias. Documento $n .^{\circ}$ 6. Educación para el trabajo y el desarrollo humano. Ministerio de Educación Nacional. https://www.mineducacion.gov. co/1759/articles-157085_archivo_pdf.pdf 
Neri, J. y Hernández, C. (2019). Competencias clave en el ámbito laboralAnálisis de la percepción estudiantil del área de ingeniería. Remai: Revista Multidisciplinar de Avances de Investigación, 5(1), 30-39.

Pedroza, R. (2018). La universidad 4.0 con currículo inteligente 1.0 en la cuarta revolución industrial. Revista Iberoamericana para la Investigación y el Desarrollo Educativo, 9(17). https://doi.org/10.23913/ ride.v9i17.377

Pérez, C. (2004). Revoluciones tecnológicas y capital financiero: La dinámica de las grandes burbujas financieras y las épocas de bonanza. Siglo XXI Editores.

Pérez, N. (2016). El SENA a la vanguardia de la cuarta revolución industrial. Revista Finnova, 2(4), 35-50.

Rentería, J., Hincapié, E., Hincapié, S., y Rojas, I. (2019). Coordenadas curriculares para la educación superior en Colombia: Objetivos de Desarrollo Sostenible (ODS), Industria 4.0 y grupos educativos. En E, Serna (ed.), Revolución en la formación y la capacitación para el siglo XXI (pp. 870-876). Editorial Instituto Antioqueño de Investigación.

Rivera, J. (2016). Los retos de la formación profesional: la formación profesional dual y la economía del conocimiento. Revista Internacional de Organizaciones, (17), 141-168.

Rojas Arenas, I. D., Vélez Castañeda, C. K., Durango Marín, J. A., Díaz Peláez, A. y Rodríguez Álvarez, A. F. (2020). Percepción del proceso de formación por competencias y su relación con las prácticas empresariales: Un caso de estudio. Revista Virtual Universidad Católica del Norte, 60, 46-68. https://doi.org/10.35575/rvucn.n60a4

Ronquillo, L., Cabrera, C., y Barberán, J. (2019). Competencias profesionales: desafíos en el proceso de formación profesional. Opuntia Brava, 11, 1-12. https://doi.org/10.35195/ob.v11iEspecial.653

Salcines, I., González, N., Ramírez, A., y Martínez, L. (2018). Validación de la escala de autopercepción de competencias transversales y profesionales de estudiantes de educación superior. Profesorado: Revista de currículum y formación del profesorado, 22(3), 31-51. https:// 10.30827/ profesorado.v22i3.7989 
Samanes, B y Martínez, P. (2018). Revolución 4.0, Competencias, educación y orientación. Revista Digital de Investigación en Docencia Universitaria, 12(2), 4-34. https://doi.org/10.19083/ridu.2018.831

Schlünzen Junior, K., Moriya Schlünzen, E. T., Zaduski, J. C. D., Ponzio, S. G. y Lopes da Silva, M. C. L. (2019). Educação Integral, Híbrida e Inclusiva na perspectiva Construcionista, Contextualizada e Significativa (CCS). Revista Interamericana de Investigación, Educación y Pedagogía (RIIEP), 13(1), 157-175. https://doi.org/10.15332/25005421/5465

Valdivieso, M., Montoya, C., y Martínez, P. (2018). Estudio de percepción de las metodologías de enseñanza-aprendizaje en un postgrado de ingeniería. Revista Interamericana de Investigación, Educación y Pedagogía (RIIEP), 12(2), 15-39. https://doi.org/10.15332/25005421.5016

Vera, M., Álava, S., y Basurto, G. (2019). El desempeño profesional: bases de la formación de calidad. Opuntia Brava, 11(1), 224-234. https://doi. org/10.35195/ob.v11i1.716

Zapata, J. (2015). El modelo y enfoque de formación por competencias en la Educación Superior: apuntes sobre sus fortalezas y debilidades. Revista Academia y Virtualidad, 8(2), 24-33.

Zúñiga, A., Leitón, R. y Naranjo, J. (2014). Del sistema educativo tradicional hacia la formación por competencias: una mirada a los procesos de enseñanza aprendizaje de las ciencias en la educación secundaria de Mendoza Argentina y San José de Costa Rica. Revista Eureka, 11(2), 145-159.

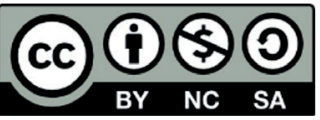

